\section{JTI}

JOURNAL OF

TRAUMA AND INJURY
Received: July 23, 2018

Revised: September 19, 2018

Accepted: September 26, 2018

\section{Correspondence to}

Sung-Hyuk Choi, M.D., Ph.D. Department of Emergency Medicine, Korea University Medical Center, Guro Hospital, 148 Gurodong-gil, Guro-gu, Seoul 08308, Korea

Tel: +82-2-2626-3283

Fax: +82-2-2626-1562

E-mail:kuedchoi@korea.ac.kr

\title{
Significance of Biomarkers as a Predictive Factor for Post-Traumatic Sepsis
}

\author{
Kyung-Wuk Lee, M.D. ${ }^{1}$, Sung-Hyuk Choi, M.D., Ph.D. ${ }^{1}$, Young-Hoon Yoon, M.D. ${ }^{1}$, \\ Jung-Youn Kim, M.D. ${ }^{1}$, Young-Duck Cho, M.D. ${ }^{1}$, Han-Jin Cho, M.D. ${ }^{1}$, \\ Sung-Jun Park, M.D. ${ }^{2}$ \\ ${ }^{1}$ Department of Emergency Medicine, College of Medicine, Korea University, Seoul, Korea \\ ${ }^{2}$ Department of Emergency Medicine, Korean Armed Forces Capital Hospital, Seongnam, \\ Korea
}

Purpose: Many traumatic patients die from sepsis and multiple organ failure. Early recognition of post-traumatic sepsis in traumatic patients will help improve the prognosis. Recently, procalcitonin (PCT), macrophage migration inhibitory factor (MIF), and lactic acid have emerged as predictive factors. Our study aims to explore the significance of PCT, MIF and lactic acid as a predictor of posttraumatic-sepsis in trauma patients.

Methods: This study was conducted on prospective observational study patients who visited an emergency medical center in a university hospital from March 2014 to February 2016. We measured the white blood cells, c-reactive protein (CRP), lactic acid, PCT, and MIF with serum taken from the patient's blood within 1 hour of the occurrence of the trauma. The definition of post-traumatic sepsis was defined as being part of systemic inflammation response syndrome criteria with infections within a week.

Results: A total of 132 patients were analyzed, wherein 74 patients were included in the low injury severity score (ISS) group (ISS $<15$ ) and 58 patients were included in the high ISS group (ISS 215 ). The mean PCT, MIF, and lactic acid levels were higher in the high ISS group $(p<0.05)$. Meanwhile, 38 patients were included in the early sepsis group and 94 patients were included in the non-sepsis group. The mean MIF levels were higher in the sepsis group than the non-sepsis group $(p<0.05)$ and there were no significant differences in the initial CRP, lactic acid, and PCT levels in these two groups.

Conclusions: MIF may be considered as a predictive factor for sepsis in trauma patients.

Keywords: Injury; Sepsis; Macrophage migration-inhibitory factors; Procalcitonin; Lactic acid 


\section{INTRODUCTION}

The mortality rate due to trauma shows three peaks over time after the injury. The first peak is immediate death after the injury. The cause of this immediate death is invariably due to the damage to the brain, brain stem, spinal cord, heart, or major blood vessels. The second peak is premature death, which occurs within hours of the injury. This premature death usually occurs by severe internal bleeding in the head, respiratory system, and abdominal cavity, or by multiple injuries resulting in excessive bleeding. Most of these injuries can be treated with the current medical techniques, but the time interval between the time of injury and the time of treatment play a decisive role in the recovery of the patient. The third peak is late death, which occurs days or weeks after the injury. Nearly $80 \%$ of these late deaths are due to sepsis or multiple organ failure [1].

When the patient is in the third peak of trauma deaths, immune responses in vivo are inhibited. This makes the body more susceptible to infection, thus leading to sepsis, multiple organ failure, and eventually death [2]. Sepsis, which is the host's response to these infections, is a major cause of morbidity and mortality worldwide. According to recent studies, 18 million cases of sepsis occur worldwide, and 30\% of them die [3]. Because of high mortality due to sepsis, prediction of post-traumatic sepsis during the early stage of trauma is believed to reduce mortality rate. Therefore, the predictive factors for sepsis in trauma patients are very important in the early stage of trauma $[4,5]$. However, there were few studies of biomarkers as the predictive factors for post-traumatic sepsis, which are different from studies of the risk factors for sepsis in trauma patients. Recently, procalacitonin (PCT), macrophage migration inhibitory factor (MIF), and lactic acid have been cited as the cause of posttraumatic-sepsis $[5,6]$. Nevertheless, there are only a few studies conducted on these factors.

Therefore, the objective of this study is to identify the significance of biomarkers, including white blood cell (WBC), c-reactive protein (CRP), PCT, lactic acid, and MIF, which are predictive factors for sepsis in Korean severe trauma patients.

\section{METHODS}

This study was conducted on patients (15 years or older) with an injury severity score (ISS) of 10 or higher who visited the emergency medical center in Korea University Guro Hospital from March 2014 to February 2016. The patients who were clearly infected at the time of arrival at the emergency medical center or who died within 72 hours of arrival were excluded from the study. Therefore, among 22,350 patients with trauma who visited the emergency medical center, the study was conducted on 169 patients (15 years or older) with an ISS of 10 or higher, who had no apparent infection at the time of hospital visit and survival more than 72 hours. Among them, 37 patients were excluded due to loss of data, etc., and the remaining 132 patients were included. The emergency center staff collected blood samples immediately upon arrival of the patient to the hospital, and informed consent was obtained from the patient or guardian to explain the purpose of the study. The study protocol and informed consent documents were reviewed and approved at the Korea University Guro Hospital (Institutional Review Board No. 13253).

The subjects were classified into two groups based on the ISS, namely, the high ISS group with 15 points or more and the low ISS group with below 15 points. As basic data, the patients' age, gender, heart rate per minute, blood pressure, respiratory rate per minute, lactic acid, PCT, MIF, and Glasgow coma scale (GCS) were measured in each group. In addition, the patients were classified into sepsis group and non-sepsis group to examine the usefulness of WBC, CRP, PCT, MIF, and lactic acid as predictors of sepsis of trauma patients.

Sepsis is life-threatening systemic symptoms arising from infection, which satisfy at least two criteria of the criteria for the systemic inflammatory response syndrome (SIRS). The infection referred herein is limited to a case in which the source of infection is clinically confirmed, such as pneumonia, urinary tract infection, wound infection, or positive blood culture within a week $[7,8]$.

All statistical analyses were performed by using the SPSS version 13.0 program (SPSS Inc., Chicago, IL, USA). The mean and normal distribution calculations, $t$-test, chi-square test, and correlation analysis were performed. 


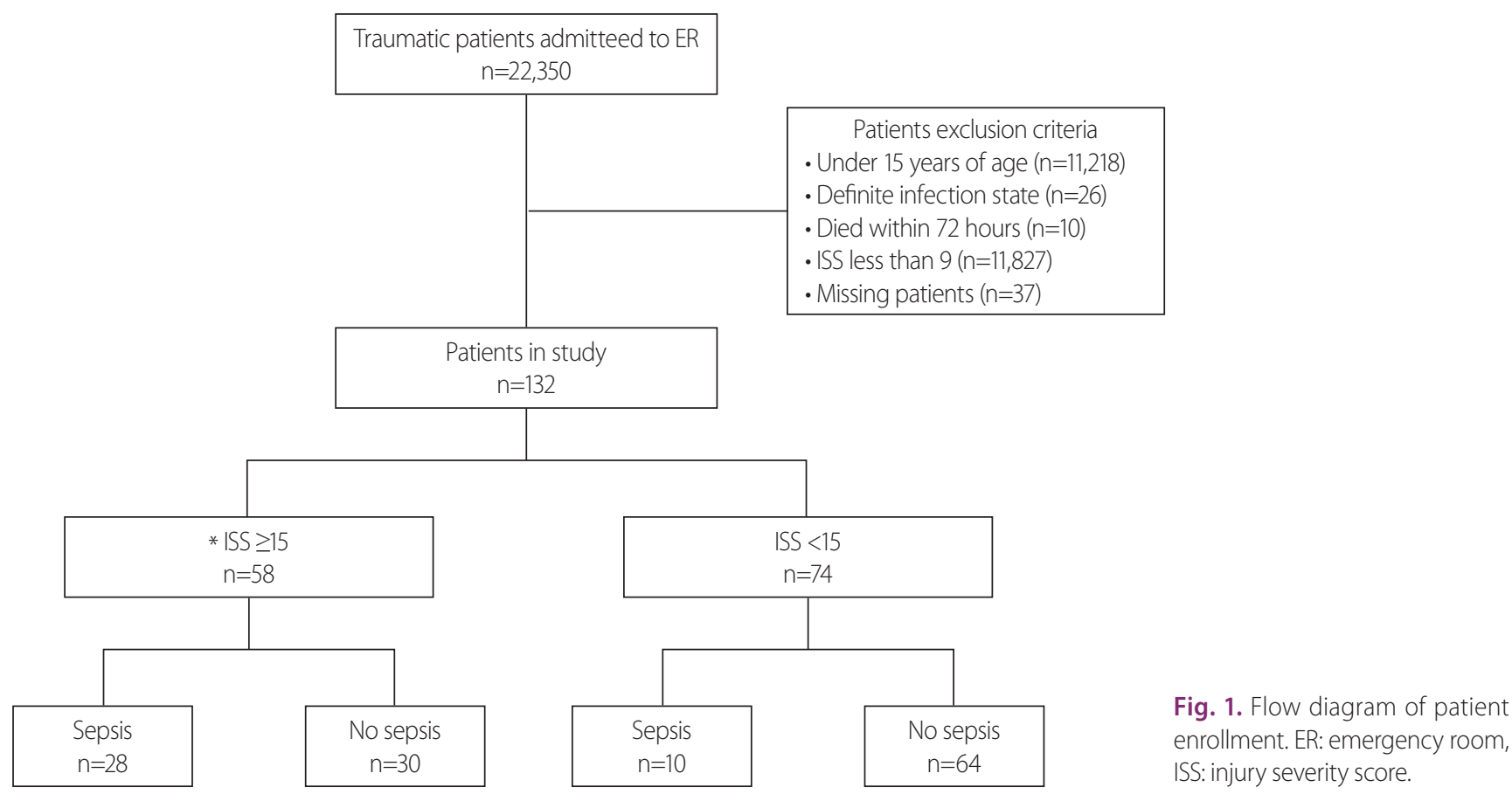

The significance probability was determined to be statistically significant when the $p$-value was below 0.05 . The data were expressed as mean \pm standard deviation.

\section{RESULTS}

\section{Characteristics of the subjects}

A total of 22,350 patients visited the emergency medical center during the period and 132 subjects were finally selected as the study subjects (Fig. 1). Fifty-eight subjects with an ISS of 15 points or more were classified into the high ISS group, and among them, 28 subjects and 30 subjects were classified into the sepsis group and non-sepsis group, respectively. In the low ISS group with an ISS of below 15, 74 patients were included, of whom 10 subjects and 64 subjects were classified into the sepsis group and non-sepsis group, respectively. The sepsis subjects accounted for $48.3 \%$ in the high ISS group and $13.5 \%$ in the low ISS group, thereby indicating a high incidence of sepsis in the high ISS group. There were no statistically significant differences in gender, age, heart rate, body temperature and respiratory rate between the high and low ISS groups. However, the systolic/diastolic blood pressures and GCS were significantly lower in the high ISS group than in the low ISS group, and lactic acid, PCT and MIF were also significantly higher in the higher ISS group than in the low ISS group (Table 1).

\section{Relation between the severity of trauma and PCT, lactic acid, and MIF}

MIF, lactic acid, and PCT levels were higher in patients with an ISS of 15 or higher than in patients with an ISS below 15. The differences in MIF, lactic acid and PCT were statistically significant, although both groups showed higher levels of MIF, lactic acid, and PCT compared with healthy persons having MIF, lactic acid and PCT levels within a normal range (Fig. 2). Simple correlation analysis was performed in order to determine the linear relationship among the ISS which is an index assessing the degree of trauma, PCT, and MIF. The Pearson correlation coefficient with ISS was 0.207 for PCT and 0.141 for MIF, and both PCT and MIF showed a weak positive linear relationship with the ISS (Fig. 3).

\section{Comparison between post-traumatic sepsis patients and non-sepsis patients}

Among the total of 132 patients who participated in the 
Table 1. Demographic data in severe trauma patients

\begin{tabular}{|c|c|c|c|}
\hline & High ISS (ISS $\geq 15)(n=58)$ & Low ISS (ISS <15) $(n=74)$ & $p$-value \\
\hline Male/female & $54 / 4$ & $58 / 16$ & 0.1 \\
\hline Age (years) & $49.1 \pm 7.9$ & $47.4 \pm 9.5$ & 0.704 \\
\hline HR (bpm) & $86.0 \pm 11.0$ & $85.2 \pm 6.8$ & 0.87 \\
\hline $\mathrm{BT}\left({ }^{\circ} \mathrm{C}\right)$ & $36.1 \pm 0.4$ & $36.5 \pm 0.2$ & $0.047^{\mathrm{a}}$ \\
\hline $\mathrm{SBP}(\mathrm{mmHg})$ & $110.3 \pm 21.0$ & $130.3 \pm 15.3$ & $0.03^{\mathrm{a}}$ \\
\hline $\mathrm{DBP}(\mathrm{mmHg})$ & $63.3 \pm 15.3$ & $80.1 \pm 9.2$ & $0.01^{\mathrm{a}}$ \\
\hline RR (breaths/min) & $21.7 \pm 3.2$ & $22.0 \pm 1.6$ & 0.727 \\
\hline Lactic acid (mmol/L) & $4.25 \pm 1.4$ & $2.25 \pm 1.1$ & $<0.01^{\mathrm{a}}$ \\
\hline$P C T^{b}(n g / m L)$ & $0.14 \pm 0.12$ & $0.085 \pm 0.09$ & $<0.01^{\mathrm{a}}$ \\
\hline $\mathrm{MIF}^{\mathrm{C}}(\mathrm{pg} / \mathrm{mL})$ & $2,633 \pm 680$ & $1,460 \pm 710$ & $<0.01^{\mathrm{a}}$ \\
\hline GCS & $12.4 \pm 2.0$ & $14.9 \pm 0.1$ & $<0.01^{\mathrm{a}}$ \\
\hline ISS & $30.2 \pm 8.0$ & $8.6 \pm 0.1$ & $<0.01^{\mathrm{a}}$ \\
\hline Sepsis & 30/58 (48.3) & $10 / 74(13.5)$ & $<0.01^{\mathrm{a}}$ \\
\hline
\end{tabular}

Values are presented as number (\%) or mean \pm SD.

ISS: Injury severity score, HR: heart rate, BT: body temperature, SBP: systolic blood pressure, DBP: diastolic blood pressure, RR: respiratory rate, PCT: procalcitonin, MIF: macrophage migration inhibitory factor, GCS: Glascow coma scale, SD: standard deviation.

There was significant statistical difference from other groups ( $p<0.05)$. Mean \pm SD paired $t$-test, chi-square test (for comparison of sex, existence of sepsis).

${ }^{b}$ Normal range of healthy human: 0-0.046 ng/mL.

${ }^{c}$ Cut-off range of healthy volunteers: $585 \pm 485 \mathrm{pg} / \mathrm{mL}$.

Concentration of MIF

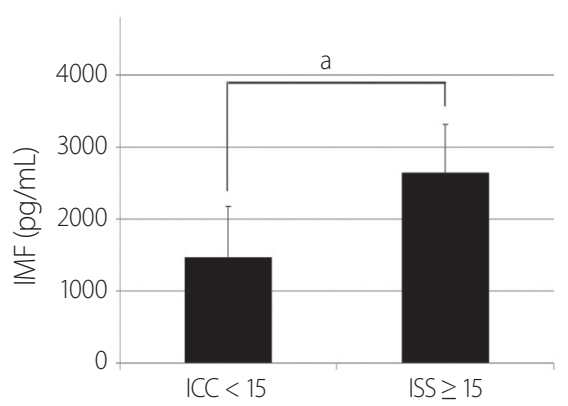

Concentration of PCT

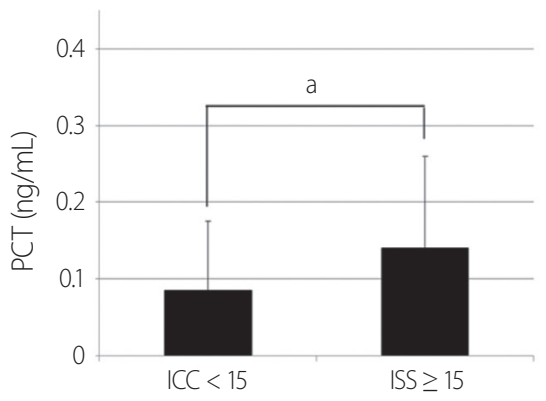

Concentration of lactic acid

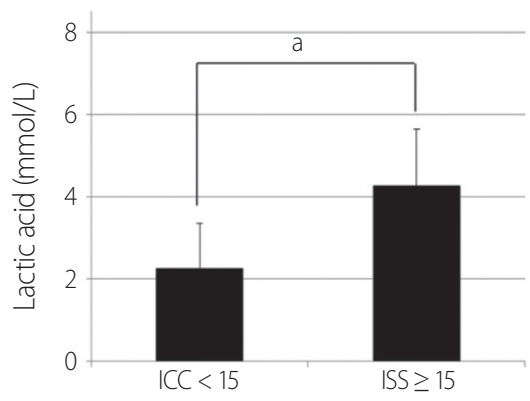

Fig. 2. Level of MIF, PCT, lactic acid in the high ISS group and low ISS group. The data are presented as mean \pm standard deviation paired t-test. MIF: macrophage migration inhibitory factor, PCT: procalcitonin, ISS: injury severity score. ${ }^{a} p<0.05$.

study, 38 patients had sepsis, while 94 patients did not have sepsis. The sepsis group and the non-sepsis group were different from each other in ISS and MIF, but no differences were found between sex, age, WBC, CRP, PCT, and lactic acid in both groups. The ISS, CRP, PCT, and lactic acid levels were the higher in the sepsis group than in the non-sepsis group, however, the ISS and MIF levels were higher with a statistical significance in the sepsis group (Table 2).

\section{DISCUSSION}

Sepsis in trauma patients is one of the major complications requiring long-term hospitalization. Around 1.5 million people in North America and 1.5 million people in North Europe are reported to experience sepsis and septic shock annually, thereby showing a mortality rate of approximately $35 \%$ to $50 \%$ [9]. Fast and reliable diagnostic tools are needed; therefore, MIF and PCT, which are 

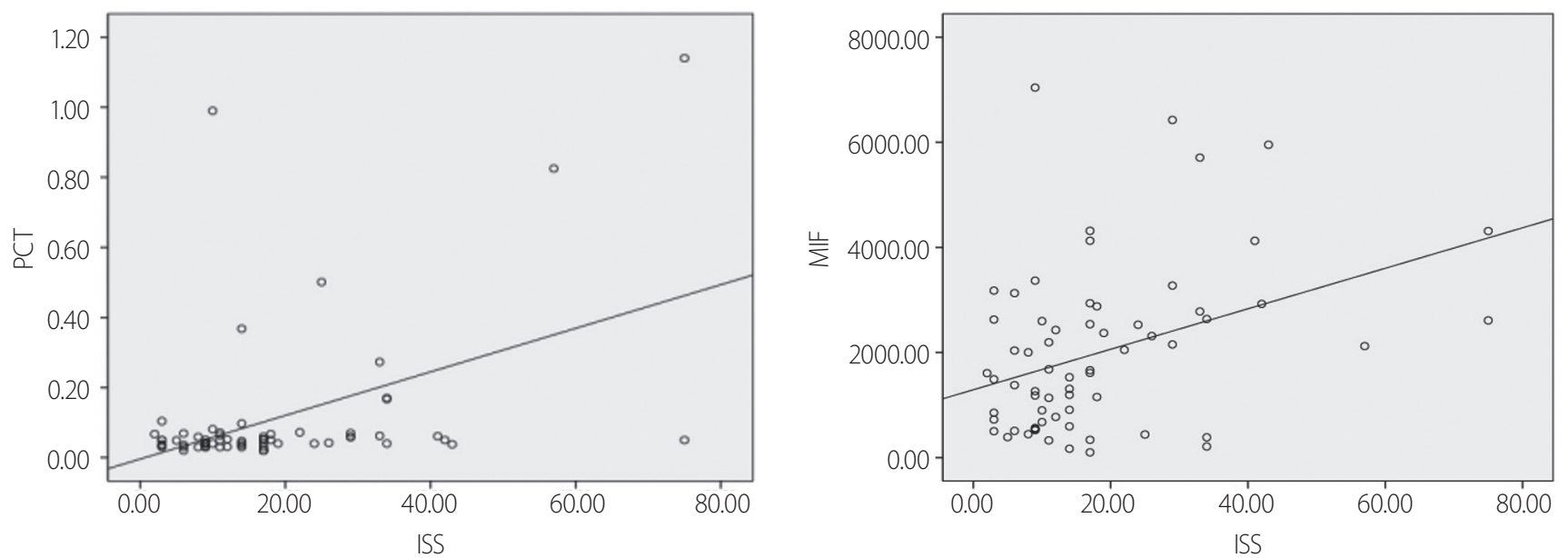

Fig. 3. Simple correlation analysis was performed in order to determine the linear relationship among the ISS, which is an index assessing the degree of trauma, PCT, and MIF. The Pearson correlation coefficient with ISS were 0.207 for PCT and 0.141 for MIF, and both PCT and MIF showed a weak positive linear relationship with the ISS. $p<0.05$. ISS: injury severity score, PCT: procalcitonin, MIF: macrophage migration inhibitory factor.

Table 2. Factors affecting post traumatic sepsis

\begin{tabular}{|c|c|c|c|}
\hline & With early sepsis $(n=38)$ & Without early sepsis $(n=94)$ & $p$-value \\
\hline Male/female & $34 / 04$ & $78 / 16$ & 0.51 \\
\hline Age (years) & $50.3 \pm 9.3$ & $47.3 \pm 8.6$ & 0.52 \\
\hline ISS & $24.7 \pm 6.6$ & $15.4 \pm 6.7$ & $0.02^{\mathrm{a}}$ \\
\hline $\mathrm{WBC}^{\mathrm{b}}\left(\mathrm{x} 10^{3}\right.$ cells/mL) & $11.0 \pm 2.5$ & $11.2 \pm 2.3$ & 0.854 \\
\hline $\mathrm{CRP}^{\complement}(\mathrm{mg} / \mathrm{L})$ & $3.9 \pm 4.3$ & $2.8 \pm 3.8$ & 0.624 \\
\hline$P C T^{d}(n g / m L)$ & $0.17 \pm 0.14$ & $0.08 \pm 0.08$ & 0.219 \\
\hline $\operatorname{MIF}^{e}(p g / m L)$ & $2,981.7 \pm 686.1$ & 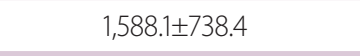 & $<0.01^{\mathrm{a}}$ \\
\hline Lactic acid $(\mathrm{mmol} / \mathrm{L})$ & $3.9 \pm 1.2$ & $3.1 \pm 1.3$ & 0.26 \\
\hline
\end{tabular}

Values are presented as number or mean \pm SD.

ISS: Injury severity score, WBC: white blood cell, CRP: c-reactive protein, PCT: procalcitonin, MIF: macrophage migration inhibitory factor, SD: standard deviation.

There was significant statistical difference from other groups $(p<0.05)$. Mean \pm SD paired $t$-test, chi-square test (for comparison of sex).

${ }^{b}$ Normal range of healthy human: $4.5-11 \times 10^{3} \mathrm{cell} / \mathrm{sL}$.

'Normal range of healthy human: 0-5 mg/L.

${ }^{d}$ Normal range of healthy human: $0-0.046 \mathrm{ng} / \mathrm{mL}$.

${ }^{e}$ Cut-off range of healthy volunteers: $585 \pm 485 \mathrm{pg} / \mathrm{mL}$.

fNormal range of healthy human: $0.5-2.2 \mathrm{mmol} / \mathrm{L}$.

produced and secreted according to the body changes of the trauma patients, have been suggested as early diagnostic indicators of sepsis of trauma patients. Recently, there have been studies on the usefulness of MIF and PCT as prognostic factors in trauma patients $[5,6]$. However, this study is meaningful in that no attempt has been made to directly compare these factors as predictors of sepsis in the early stages of severe trauma patients.
Our study shows that MIF, PCT, and lactic acid levels were all higher in patients with an ISS of 15 or higher than in patients with an ISS below 15 (Fig. 2). And Pearson correlation coefficient representing the correlations with ISS were 0.207 in PCT and 0.141 in MIF (Fig. 3). In other words, both MIF and PCT had a positive correlation with the degree of trauma, and the PCT was more statistically significant. Thus, the severity of the trauma is related to 
the PCT, MIF, lactic acid. As in our study, other studies have also shown an increase in PCT as trauma severity increases [10]. Our study shows that ISS, CRP, PCT, MIF, and lactic acid were higher in the post-traumatic early sepsis group than in the non-sepsis group, however, only ISS and MIF had statistically significant differences at the $p$-value $<0.05$ (Table 2).

PCT is estimated to present a diagnostic index that can more accurately predict sepsis and it is being studied as an indicator to distinguish non-infectious causes that may result in SIRS from infectious causes. PCT begins to rise within 6 hours to 12 hours of infection and it is halved a day when the infection is controlled by the host's immune system and antibiotics [11]. In this study, PCT was not statistically significant as an early predictor of sepsis in trauma patients in this study. It is difficult to predict posttraumatic-sepsis through PCT, since PCT is assumed to be increased in the event of an infection and begins to rise within 6 hours to 12 hours of infection, making it impossible to predict the occurrence of the infection later [10].

MIF is another factor that is newly emerging for predicting the inflammatory response in patients with trauma combined with sepsis. It acts as a chemokine-like cytokine that recruits WBC and migrates them to infected and inflamed areas $[12,13]$. MIF is stored in the pituitary T-cells and macrophages, and it responds to stress-like stimuli. The major roles of MIF include macrophage regulation, leukocyte immune function regulation, endocrine function, and inhibition of glucocorticoid immunosuppression and anti-inflammatory action. MIF plays an important role in acute inflammatory diseases, such as sepsis shock, rheumatic disease, interstitial lung disease, glomerulonephritis, and inflammatory bowel disease. Unlike other biomarkers that represent hyperinflammation, MIF reflects not only hyperinflammation but also immune suppression [14-16]. In this study, the significance as a predictor of sepsis in patients with severe trauma was higher in MIF than in PCT. Because of the absence of infection in the early stage of severe trauma, it is reasonable to assume that the immune suppression status at the early stage of trauma is likely to develop into an infection such as sepsis later. Thus, reflecting this immune suppression status, MIF is considered to be one of the predictors of sepsis in severe trauma patients. And another study reported that MIF is a useful predictor of early sepsis in trauma patients based on the trauma mechanism, revised trauma score, survival rate, length of stay in the intensive care unit [6]. This supports the findings of our study, in which MIF was statistically significant as a predictor of early sepsis in trauma patients.

The lactic acid is the final product of anaerobic action. It is produced by pyruvate metabolism and its degree of production is determined by the redox state of cytoplasm. In a hypoxic state, oxidative phosphorylation of mitochondria is difficult and pyruvic acid is accumulated, which converts to lactic acid [17]. In patients with sepsis due to severe trauma, if the oxygen supply is not enough and cannot keep up with the required oxygen consumption, it results in hypoxia in the overall tissues, and lactic acid is accumulated as a product of anaerobic metabolism. Therefore, lactic acid is known as an index reflecting tissue hypoxia $[18,19]$. For this reason, lactic acid has attracted much attention as a predictor of sepsis. However, another study reported the lactic acid that was measured in the early emergency room stay in severe trauma patients was not statistically significant between the survival and non-survival groups, thereby indicating that it is not appropriate as a predictor of mortality. When considering the high proportion of death by sepsis in severe trauma deaths, though it is different from the present study that used lactic acid as a predictor of sepsis, lactic acid is not considered as an appropriate predictor of trauma death or sepsis. Also, the results of our study were not statistically significant as an early predictor of sepsis in severe trauma patients. It is presumed that lactic acid reflects sepsis but is not a predictor of sepsis.

The MIF level of the sepsis group was statistically significant and it also was higher than that of the non-sepsis group in the test for blood that was collected within 1 hour. That is to say, sepsis is estimated to be caused by various causes, such as hyperinflammation and immunosuppression, therefore, MIF can be used as a factor to evaluate the overall conditions of the patient unlike PCT, such as inflammation or decline in immunity, and sepsis after the initial stage, and it may be useful for the early diagnosis of trauma patients. However, further studies are deemed necessary for the mechanism of the MIF elevation, and the changes in MIF with time after the trauma 
should be further investigated through animal experiments.

This study has some limitations. Firstly, this study has devised means of determining the effectiveness of biomarkers as a predictor of post-traumatic sepsis in trauma patients; however, only a small number of patients participated in this study, and therefore various factors known to affect the occurrence of post-traumatic sepsis, such as age, ISS, blood transfusion, and types of trauma, could not be excluded. Secondly, MIF is presented as a significant biomarker in predicting post-traumatic sepsis. But more data are needed to determine the cut-off value. Thirdly, ISS of 15 points was used to measure the severity of the trauma since there were a few patients enrolled in the study, therefore, the research using of 25 points may be needed later. And lastly, in this study various biomarkers were used for early diagnosis of sepsis. But we do not know how treatments and prognosis would be different. It would be great if studies were conducted on the effects of early treatments with early diagnosis, such as the prompt use of antibiotics, small amounts of blood transfusions, and prompt operation, etc.

\section{CONCLUSION}

This study supports the previous studies that MIF is useful as a predictor of early sepsis in trauma patients, but the usefulness of PCT as a predictor of early sepsis is somewhat different from that of the previous studies. For this reason, further research is deemed necessary in a larger number of patients.

\section{ACKNOWLEDGEMENTS}

This study was partially supported by Korea University Grant and Basic Science Research Program through the National Research Foundation of Korea (NRF) funded by the Ministry of Education, Science and Technology (R1804431).

\section{REFERENCES}

1. Trunkey DD. Trauma. Accidental and intentional injuries account for more years of life lost in the U.S. than cancer and heart disease. Among the prescribed remedies are improved preventive efforts, speedier surgery and further research. Sci Am 1983;249:28-35.

2. Bernhagen J, Calandra T, Bucala R. Regulation of the immune response by macrophage migration inhibitory factor: biological and structural features. J Mol Med (Berl) 1998;76:151-61.

3. Slade E, Tamber PS, Vincent JL. The Surviving Sepsis Campaign: raising awareness to reduce mortality. Crit Care 2003;7:1-2.

4. Park JH, Choi SH, Yoon YH, Park SJ, Kim JY, Cho HJ. Risk factors for sepsis in Korean trauma patients. Eur J Trauma Emerg Surg 2016;42:453-8.

5. Rajkumari N, Mathur P, Sharma S, Gupta B, Bhoi S, Misra MC. Procalcitonin as a predictor of sepsis and outcome in severe trauma patients: a prospective study. J Lab Physicians 2013;5:100-8.

6. Cho YD, Choi SH, Kim JY, Park SJ, Yoon YH, Cho HJ, et al. Macrophage migration inhibitory factor levels correlate with an infection in trauma patients. Ulus Travma Acil Cerrahi Derg 2017;23:193-8.

7. Bone RC. Let's agree on terminology: definitions of sepsis. Crit Care Med 1991;19:973-6.

8. Annane D, Bellissant E, Cavaillon JM. Septic shock. Lancet 2005;365:63-78

9. Becker JU, Theodosis C, Jacob ST, Wira CR, Groce NE. Surviving sepsis in low-income and middle-income countries: new directions for care and research. Lancet Infect Dis 2009;9:57782.

10. Mimoz O, Benoist JF, Edouard AR, Assicot M, Bohuon C, Samii K. Procalcitonin and C-reactive protein during the early posttraumatic systemic inflammatory response syndrome. Intensive Care Med 1998;24:185-8.

11. Becker KL, Nylén ES, White JC, Müller B, Snider RH Jr. Clinical review of 167: procalcitonin and the calcitonin gene family of peptides in inflammation, infection, and sepsis: a journey from calcitonin back to its precursors. J Clin Endocrinol Metab 2004;89:1512-25.

12. Bernhagen J, Krohn R, Lue H, Gregory JL, Zernecke A, Koenen $\mathrm{RR}$, et al. MIF is a noncognate ligand of CXC chemokine receptors in inflammatory and atherogenic cell recruitment. Nat Med 2007;13:587-96. 
13. Schober A, Bernhagen J, Weber C. Chemokine-like functions of MIF in atherosclerosis. J Mol Med (Berl) 2008;86:761-70.

14. Calandra T, Roger T. Macrophage migration inhibitory factor: a regulator of innate immunity. Nat Rev Immunol 2003;3:791800.

15. Froidevaux C, Roger T, Martin C, Glauser MP, Calandra T. Macrophage migration inhibitory factor and innate immune responses to bacterial infections. Crit Care Med 2001;29(7 Sup$\mathrm{pl}): S 13-5$.

16. Bozza M, Satoskar AR, Lin G, Lu B, Humbles AA, Gerard C, et al. Targeted disruption of migration inhibitory factor gene reveals its critical role in sepsis. J Exp Med 1999;189:341-6.

17. Levy B. Lactate and shock state: the metabolic view. Curr Opin Crit Care 2006;12:315-21.

18. Bakker J, Gris P, Coffernils M, Kahn RJ, Vincent JL. Serial blood lactate levels can predict the development of multiple organ failure following septic shock. Am J Surg 1996;171:221-6.

19. Shin TG, Hwang SY, Kang GH, Kim WY, Ryoo SM, Kim K, et al. Korean Shock Society septic shock registry: a preliminary report. Clin Exp Emerg Med 2017;30:146-53. 